\title{
Effect of Skin Temperature on the Cholinergic Sensitivity of the Human Eccrine Sweat Gland
}

\author{
Dana M. DIPASQUALE ${ }^{*}$, Michael J. BUONO ${ }^{*,}$, and Fred W. KOLKHORST* \\ * Department of Exercise and Nutritional Sciences, ${ }^{\dagger}$ Department of Biology, San Diego State University, \\ San Diego, CA 92182-7251, U.S.A.
}

\begin{abstract}
Although sweat gland activity is directly controlled by the central nervous system, which detects changes in core body temperature, sweat glands can also be influenced by local cutaneous thermal conditions. Objective: The present study sought to determine the effect of local skin temperature on pilocarpine-induced sweating within a range of typical skin temperatures. Methods: Thirteen subjects ( $30 \pm 6$ years; $172 \pm$ $11 \mathrm{~cm} ; 72.8 \pm 11.0 \mathrm{~kg}$ ) had forearm sweat rates measured at rest following pilocarpine iontophoresis at each of three skin temperatures in randomized order: warm $\left(T_{\text {warm }}=37.1 \pm 0.9^{\circ} \mathrm{C}\right)$, control $\left(T_{\text {con }}=32.3 \pm 1.4^{\circ} \mathrm{C}\right)$, and cool $\left(T_{\text {cool }}=\right.$ $\left.26.6 \pm 1.3^{\circ} \mathrm{C}\right) . T_{\text {skin }}$ was raised and lowered with an electric heating pad and gel ice pack, respectively. Forearm $T_{\text {skin }}$ was measured with a skin temperature probe. Pilocarpine iontophoresis was used on an approximately $7 \mathrm{~cm}^{2}$ area of the
\end{abstract}

anterior forearm to stimulate localized sweating. Following stimulation, sweat was collected from the area for 15 min with a Macroduct Sweat Collection System. Results: There was a higher sweat rate at $T_{\text {warm }}(p=0.001)$ and $T_{\text {con }}(p=0.006)$ compared to that at $T_{\text {cool }}$. However, there was no difference between the sweat rate at $T_{\text {warm }}$ and that at $T_{\text {con }}(p=0.127)$. Conclusion: These results indicated that skin temperatures below approximately $32^{\circ} \mathrm{C}$ affect local sweat production primarily by altering glandular sensitivity to the neurotransmitter, whereas skin temperatures above approximately $32^{\circ} \mathrm{C}$ predominantly affect neurotransmitter release. Furthermore, sweat glands display maximal or near maximal cholinergic sensitivity at resting skin temperature in a thermoneutral environment. [The Japanese Journal of Physiology 53: 427-430, 2003]

Key words: skin temperature, sweat glands, neurotransmitter, cholinergic sensitivity, pilocarpine iontophoresis.

\begin{abstract}
Although sweat gland activity is directly controlled by the central nervous system (CNS), which detects changes in core body temperature, sweat glands can also be influenced by local conditions. For example, an increase in local skin temperature $\left(T_{\text {skin }}\right)$ results in increased sweating. However, the mechanism by which this occurs remains unclear. Little research has been performed to answer this question, but two main possibilities have been suggested. First, an increase in local $T_{\text {skin }}$ results in a greater release of sudomotor neurotransmitter, causing a greater sweat response [1, 2]. Second, sweat glands may exhibit an enhanced sensitivity to a given neurotransmitter following a rise
\end{abstract}

in local $T_{\text {skin }}[3,4]$. Qualitative observations on two subjects tested by Ogawa [3] suggested that increasing $T_{\text {skin }}$ from approximately 23 to $36^{\circ} \mathrm{C}$ resulted in a decreased amount of pilocarpine or acetylcholine needed to induce a noticeable sweating response. Similar results were found in the same study when $T_{\text {skin }}$ was increased from 26 to 29.5 and then to $32^{\circ} \mathrm{C}$ on one subject. Follow-up work on four subjects by Ogawa and Asayama [4] indicated that an increase in $T_{\text {skin }}$ from approximately 37 to $42^{\circ} \mathrm{C}$ increased the rate of pilocarpine-induced sweat production. Although the small sample sizes prevented statistical analysis, these collective results suggested that local heating fa-

Received on June 5, 2003; accepted on November 17, 2003

Correspondence should be addressed to: Dana M. DiPasquale, Department of Movement Sciences (m/c 194), University of IllinoisChicago, 901 W. Roosevelt Rd., Chicago, IL 60608, USA. Tel: +1-312-996-7730, Fax: +1-312-996-2958, E-mail: ddipas1@uic.edu 
cilitated sweat production by increasing glandular sensitivity to the neurotransmitter.

However, the extreme $T_{\text {skin }}\left(23-42^{\circ} \mathrm{C}\right)$ used in these studies were not values typically seen [5-8] and may have affected the results. Therefore the present study sought to determine the effect of local $T_{\text {skin }}$ on pilocarpine-induced sweating within a range of typical skin temperatures.

\section{MATERIALS AND METHODS}

Thirteen subjects $(30 \pm 6$ years; $172 \pm 11 \mathrm{~cm} ; 72.8 \pm$ $11.0 \mathrm{~kg}$ ) participated in the study following their informed consent as approved by the university's institutional review board. Subjects refrained from exercise for at least $5 \mathrm{~h}$ prior to data collection and did not eat or drink during testing. Ambient temperature was approximately $25^{\circ} \mathrm{C}$ with a relative humidity of approximately $40 \%$.

Skin temperature. Subjects had forearm sweat rates measured at rest following pilocarpine iontophoresis at each of three skin temperatures in random order: warm $\left(T_{\text {warm }}=37.1 \pm 0.9^{\circ} \mathrm{C}\right)$, control $\left(T_{\text {con }}=32.3 \pm 1.4^{\circ} \mathrm{C}\right)$, and cool $\left(T_{\text {cool }}=26.6 \pm 1.3^{\circ} \mathrm{C}\right)$. $T_{\text {skin }}$ was raised and lowered approximately $5^{\circ} \mathrm{C}$ from $T_{\text {con }}$ with an electric heating pad and gel ice pack, respectively, for approximately $15-20$ min to reach and maintain the desired temperature during sweat production and collection. An elastic bandage protected the skin from direct contact with the ice pack, and a fabric cover protected it from direct contact with the heating pad. A small foam pad was placed over the thermistor to avoid direct contact with the heating/cooling element. Forearm $T_{\text {skin }}$ was measured with a skin temperature probe (YSI model 47, Yellow Springs, OH, USA).

Sweat production and collection. Pilocarpine iontophoresis was initiated after $T_{\text {skin }}$ stabilized to $\pm 0.5^{\circ} \mathrm{C}$ for $5 \mathrm{~min}$. An approximately $7-\mathrm{cm}^{2}$ area of the anterior forearm was stimulated to produce localized sweating at the site. Prior to electrode placement, the skin was abraded with an alcohol pad and sterile gauze. A razor was used to remove hair if necessary. Iontophoretic electrodes were placed on the anterior forearm, and for subsequent trials using different skin temperatures, they were placed within $3 \mathrm{~cm}$ of the original location, though not on an overlapping area. The Wescor instrument uses a $1.5 \mathrm{~mA}$ current with $0.5 \%$ pilocarpine in the each disk. This will deliver $0.10 \mathrm{mg}$ of pilocarpinium ion to each square $\mathrm{cm}$ of skin in $5 \mathrm{~min}$. Following stimulation, sweat was collected from the area for 15 min with a Macroduct Sweat Collection System (Wescor, UT,
USA).

Statistical analysis. Sweat rate at each of the three skin temperatures was compared by using a repeated measures ANOVA with an alpha level of 0.05 . Post hoc comparisons were performed with Bonferroni's test. All data are expressed as means \pm SD.

\section{RESULTS}

Although sphericity was not assumed $(p=0.04)$, the Greenhouse-Geisser adjustment for RM ANOVA indicated a difference in sweat rate between the three temperatures $(p=0.001)$. Bonferroni post hoc analysis indicated higher sweat rates at $T_{\text {warm }}(7.1 \pm 2.7$ $\left.\mathrm{g} \mathrm{m}^{-2} \mathrm{~min}^{-1} ; \quad p=0.001\right)$ and at $T_{\text {con }}(6.2 \pm 3.1$ $\left.\mathrm{g} \mathrm{m}^{-2} \mathrm{~min}^{-1} ; p=0.006\right)$ compared to $T_{\text {cool }}(3.4 \pm 1.7$ $\left.\mathrm{g} \mathrm{m}^{-2} \min ^{-1}\right)$. However, there was no difference between the sweat rate at $T_{\text {warm }}$ and that at $T_{\text {con }}(p=$ 0.127) (Fig. 1).

\section{DISCUSSION}

Previous research has indicated that a rise in $T_{\text {skin }}$ caused an increase in sweat production via greater neurotransmitter release or an increase in the cholinergic sensitivity of sweat glands [1-4]. Collectively, this research suggests that a rise in neurotransmitter release is responsible for increases in sweat production at high $T_{\text {skin }}$, though glandular sensitivity is affected at low $T_{\text {skin }}$ (Fig. 2). For example, Elizondo [1] found an increase in neurotransmitter release after warming $T_{\text {skin }}$ to $42^{\circ} \mathrm{C}$, from $37^{\circ} \mathrm{C}$. MacIntyre et al. [2] reported similar results when they increased $T_{\text {skin }}$ to $42^{\circ} \mathrm{C}$, from $36^{\circ} \mathrm{C}$. Conversely, limited published data suggested an increase in sensitivity at $T_{\text {skin }}$ of less than $36^{\circ} \mathrm{C}$ [6]; however, a later report from the same laboratory suggested that $T_{\text {skin }}$ above $37^{\circ} \mathrm{C}$ also increased sensitivity to neurotransmitter [4]. The latter observations, how-

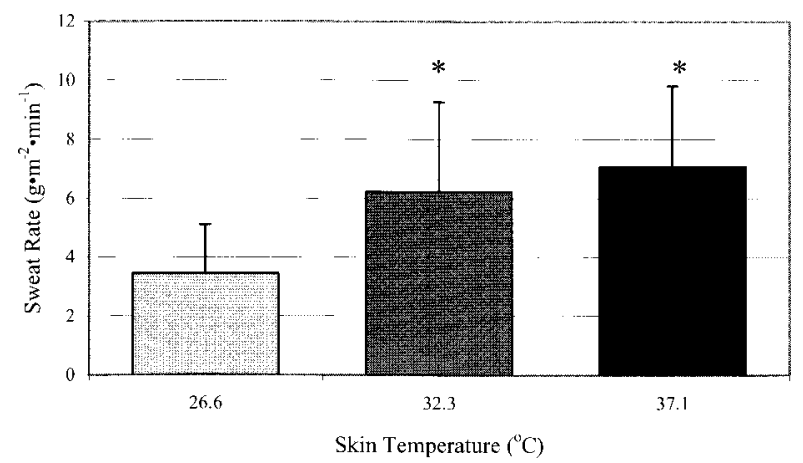

Fig. 1. Effect of $\boldsymbol{T}_{\text {skin }}$ on sweat rate. Sweat rate at $T_{\text {warm }}$ $(p=0.001)$ and at $T_{\text {con }}(p=0.006)$ was higher than that at $T_{\text {cool. }}$. There was no difference between sweat rate at $T_{\text {warm }}$ and $T_{\text {con }}(p=0.127)$. 


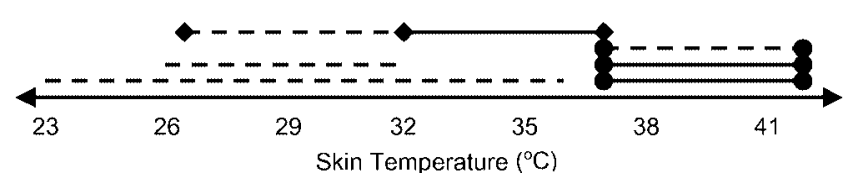

Fig. 2. The collective summary of previous research and the present study suggests that a rise in neurotransmitter release is responsible for increases in sweat production at high $T_{\text {skin }}$, while glandular sensitivity is affected at low $\boldsymbol{T}_{\text {skin. }}$. Solid lines indicate a change in neurotransmitter release caused increased sweat production; dashed lines indicate a change in glandular sensitivity. The present study is designated by the lines with diamonds while lines with circles reflect data collected during exposure to high ambient temperature.

ever, may have been the result of central mechanisms rather than peripheral ones, since ambient temperatures in this study were 35 to $41^{\circ} \mathrm{C}$. Although not reported, these high ambient temperatures may have been warm enough to increase core body temperature and initiate sweating via the CNS. Similarly, the increase in neurotransmitter releases observed by Elizondo [1] and MacIntyre et al. [2] were in ambient temperatures of 40 and $38^{\circ} \mathrm{C}$, respectively and therefore may also have been enhanced by central mechanisms.

The present study was designed to quantify the effect of $T_{\text {skin }}$ on sweat production within a typically observed range of $T_{\text {skin. }}$. Pilocarpine iontophoresis was used to stimulate sweat production with a set amount of agonist while bypassing the CNS. Because input from the CNS was constant, any change in sweat production could be attributed to the local change in $T_{\text {skin }}$.

Our findings demonstrated that within a typical range of skin temperatures, peripheral sweat rate at $T_{\text {con }}$ was decreased at $T_{\text {cool }}$ but was unaffected at $T_{\text {warm }}$. Such results indicated that cholinergic receptors on sweat glands may be less sensitive to agonists when $T_{\text {skin }}$ is below $32^{\circ} \mathrm{C}$, thereby supporting previous studies $[3,4]$. Moreover, these results suggested that an increase in sweat rate following an increase in $T_{\text {skin }}$ above $T_{\text {con }}$ is more likely a result of an increased release of sudomotor transmitter rather than increased sensitivity, also confirming previous findings $[1,2]$.

Because there was no difference in sweat rates at $T_{\text {warm }}$ and $T_{\text {con }}$, it may be possible that cholinergic sensitivity is maximal or near maximal at $T_{\text {con }}$. Although the difference in sweat rate at $T_{\text {con }}$ compared to $T_{\text {warm }}$ was not enough to be statistically significant, the relatively small $p$ value indicated that the sensitivity changes observed at cooler $T_{\text {skin }}$ did not completely disappear at warmer $T_{\text {skin. }}$. This suggests that both mechanisms are very likely acting simultaneously but contributing in different proportions. Moreover, the

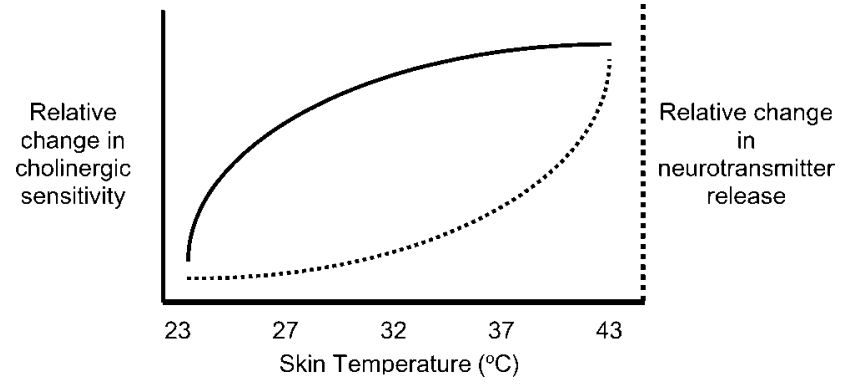

Fig. 3. Proposed model of the relative contributions of glandular sensitivity and neurotransmitter release to increases in sweat production at various $\boldsymbol{T}_{\text {skin. }}$. Both mechanisms are likely acting simultaneously, although contributing in different proportions. Results indicated that at $T_{\text {skin }}$ near $32^{\circ} \mathrm{C}$ there may be a change in the predominant contributor, either sensitivity or neurotransmitter, to changes in sweat production.

results indicated that at a $T_{\text {skin }}$ near $32^{\circ} \mathrm{C}$ there may be a change in the predominant contributor, either sensitivity or neurotransmitter release, to changes in sweat production (Fig. 3).

A limitation of the present study is that the cholinergic agonist was administered iontophoretically. Consequently, it cannot be guaranteed that the same amount of agonist reached the sweat gland in all three cutaneous thermal conditions. It is possible that an increase in cutaneous blood flow may wash out the agonist from the localized area. Another model may use the injected agonist; however, the same complications with cutaneous blood flow may arise, which is the limitation for most in vivo models. Although a skin biopsy with in vitro sweat gland stimulation may eliminate this problem, whether this is a physiologically applicable situation is questionable.

In summary, these results indicated that $T_{\text {skin }}$ below approximately $32^{\circ} \mathrm{C}$ affects local sweat production primarily by altering glandular sensitivity, whereas temperatures above approximately $32^{\circ} \mathrm{C}$ predominantly affect neurotransmitter release. Furthermore, sweat glands display maximal or near maximal cholinergic sensitivity at resting $T_{\text {skin }}$ in a thermoneutral environment. Future studies may revise the present in vivo model to ensure that the amount of agonist delivered to the sweat glands is identical in all temperature conditions.

\section{REFERENCES}

1. Elizondo RS: Local control of eccrine sweat gland function. Fed Proc 32: 1583-1587, 1973

2. Maclntyre BA, Bullard RW, Banerjee $M$, and Elizondo $R$ : Mechanism of enhancement of eccrine sweating by localized heating. J Appl Physiol 25: 255-260, 1968

3. Ogawa T: Local effect of skin temperature on threshold 
concentration of sudorific agents. J Appl Physiol 28: 18-22, 1970

4. Ogawa T and Asayama M: Quantitative analysis of the local effect of skin temperature on sweating. Jpn J Physiol 36: 417-422, 1986

5. Nadel ER, Bullard RW, and Stolwijk JAJ: Importance of skin temperature in the regulation of sweating. $\mathrm{J}$ Appl Physiol 31: 80-87, 1971

6. Livingstone SD, Nolan RW, Frim J, Reed D, and Limmer RE: A thermographic study of the effect of body composition and ambient temperature on the accuracy of mean skin temperature calculations. Eur J Appl Physiol 56: 120-125, 1987

7. Montgomery LD and Williams BA: Effect of ambient temperature on the thermal profile of the human forearm, hand, and fingers. Ann Biomed Eng 4: 209-219, 1976

8. Olsen BW and Fanger PO: The skin temperature distribution for resting man in comfort. Arch Sci Physiol 27: A385-A393, 1973 